\title{
1 THE INDUCTION OF LARVAL RESOURCE PREFERENCE IN HETEROGENEOUS HABITATS
}

2 Vrinda Ravi Kumar*, Swastika Issar ${ }^{1,2}$ and Deepa Agashe ${ }^{1 *}$

3

$4 \quad{ }^{1}$ National Centre for Biological Sciences

5 Tata Institute of Fundamental Research

6 Bellary Road, Bangalore, India 560065

7

$8{ }^{2}$ Current address:

9 Department of Zoology

10 University of Cambridge

11 Downing Street, United Kingdom

12

$13 *$ Corresponding authors:

14 vrindark@ncbs.res.in

15 dagashe@ncbs.res.in

16

17 Keywords: resource preference, diet breadth, Tribolium castaneum, habitat heterogeneity, resource experience,

18 novel resources 


\section{ABSTRACT}

20 Animals often have to evaluate and choose between multiple food sources in their habitat, and these potentially

21 complex decisions can have a large impact on their fitness. Among other factors, previous experience with an

22 alternative resource can significantly increase subsequent preference for the resource ("induction of preference").

23 Such induction of resource preference is particularly relevant in spatially or temporally heterogeneous habitats.

24 Although most mobile species - especially generalists - probably frequently encounter habitat heterogeneity, the

25 impact of preference induction on individual behaviour and fitness in heterogeneous habitats is poorly

26 understood. We analysed larval preference induction in wheat-adapted generalist red flour beetles (Tribolium

27 castaneum) under three types of habitat heterogeneity. We first analysed the induction of larval preference for

28 novel resources (other cereal flours) under temporal heterogeneity, exposing larvae to new resources during

29 development. We found that larvae preferred a new resource if they experienced it recently, but that the

30 magnitude of induction varied across resources. Interestingly, we also observed specific induction for a

31 homogenous mix of wheat and a novel resource, with larvae preferring the mix over either pure resource. To

32 analyse induction under spatial heterogeneity, we placed beetle eggs in one of two alternative resource patches

33 and tested the preference of emerged larvae. Unexpectedly, hatching into a novel resource did not always induce

34 preference. Finally, we found that induction of preference for new resources could be maladaptive for larval

35 development. Together, our work demonstrates that experience-based plasticity of larval resource choice may

36 strongly impact larval preference and fitness in heterogeneous habitats. 


\section{INTRODUCTION}

38 Insects occupy a large diversity of dietary niches, and a substantial body of work has thus focused on

39 understanding insect resource choice. Changes in resource preference can facilitate dietary niche shifts (Agosta

40 2006), potentially leading to divergence in host-associated traits (Singer \& McBride 2010). Thus far, most

41 studies of resource choice in holometabolous insects have analyzed only adult preference, since it is often well

42 correlated with larval performance (Dethier 1959; Jaenike 1978; Gripenberg et al. 2010). However, many larvae

43 also exhibit resource choice (Jermy et al. 1968; Berdegue \& Trumble 1996; Bernays \& Weiss 1996; Gamberale-

44 Stille et al. 2014) and are capable of exploring different habitat patches over smaller spatial scales (Abbott \&

45 Dukas 2016). Thus, even though adult oviposition preference often determines larval resource use, it is important

46 to study larval choice in spatially heterogeneous environments, where larval preference may also contribute

47 substantially to larval fitness. Under such conditions, it is possible that resource choice is not the sole domain of

48 the adult, and may instead be shared across stages (Wiklund 1975; Chew 1977; Berdegue et al. 1998;

49 Gamberale-Stille et al. 2014; Abbott \& Dukas 2016). However, because larval preference is relatively

50 understudied, we do not know the impact of larval preference on larval fitness in heterogeneous environments.

51

52 What factors shape larval preference in heterogeneous habitats? A large body of work suggests that prior

53 experience with alternative resources can strongly shape larval preference. Many Lepidopteran larvae show

54 “induction of preference”: an increased preference for host plants that they have experienced previously (Jermy

55 et al. 1968; Bernays \& Weiss 1996; Carlsson et al. 1999; del Campo et al. 2001; Henniges-Janssen et al. 2014;

56 Soler et al. 2012; Gretes et al. 2016). A particularly striking example is the induction of preference in Manduca

57 sexta. While naïve larvae can feed and survive on many host plants, larvae that feed on solanaceous foliage

58 become specialized and reject other hosts (Schoonhoven 1967; Yamamoto 1974; del Campo \& Renwick 2000).

59 Similar induction of preference also occurs in some non-Lepidopteran insects (Phillips 1977). Although

60 preference induction thus seems to be common across insects, little is known about its occurrence in natural

61 conditions and its impact on insect fitness. Previous studies suggest that the induction of preference could lead to

62 increased acceptability of the inducing host, improving subsequent insect performance on it through higher 
63 feeding and/or physiological acclimation (Schoonhoven \& Meerman 1978; Scriber 1979; Karowe 1989; Agrawal

64 et al. 2002). However, these studies focus on mechanistic aspects of experience-mediated changes in food

65 acceptability and digestive efficiency, leaving fundamental questions about the induction of preference under

66 resource heterogeneity unanswered. For instance, we do not know the impact of experience with temporally or

67 spatially heterogeneous resources on larval preference, and consequently on larval resource use. This is

68 particularly important since most animals probably encounter significant habitat heterogeneity over their

69 lifespan. Larval movement in a heterogeneous habitat could result in larvae simultaneously or sequentially

70 experiencing different resources, but how does the induction of preference operate in such cases? For instance,

71 does the duration of exposure to a resource impact preference, or is preference determined during a specific

72 developmental period? Does the magnitude of induction vary depending on available resources? Does induction

73 by the natal resource dictate larval resource choice in a patchy habitat (as is often assumed), or does the presence

74 of alternative resources decrease the impact of the natal experience on larval choice? Finally, is the induction of

75 larval preference adaptive?

77 To address these questions, we tested the occurrence and strength of induction of larval resource preference in a

78 generalist insect pest of stored grain flour - the red flour beetle Tribolium castaneum (Sokoloff 1974; Ziegler

79 1976). Flour beetles are strong dispersers and frequently colonize grain warehouses (Naylor 1961; Hagstrum \&

80 Gilbert 1976; Ziegler 1976; Ziegler 1977). Resource availability within storage warehouses fluctuates over time,

81 and beetles often move across flour patches (Ziegler 1976; Campbell \& Hagstrum 2002), experiencing several

82 resources in their lifetime and potentially exerting resource choice. Prior work suggests that T. castaneum larval

83 preference can oppose adult resource preference: given a choice between the ancestral wheat resource and a

84 suboptimal novel resource (corn), T. castaneum larvae favoured wheat, while adult females preferentially

85 oviposited in corn (Agashe \& Bolnick 2012). At high density, larvae raised in a heterogenous wheat-corn patchy

86 habitat also preferred corn more strongly than larvae from a homogenous wheat habitat (Parent et al. 2014),

87 suggesting that prior experience in a heterogeneous habitat alters larval resource choice in the face of

88 competition. Thus, T. castaneum larval choice may determine larval resource use in heterogeneous 
89 environments. Here, we examined the role of larval choice and the impacts of resource heterogeneity on larval

90 resource preference and fitness in T. castaneum. We first tested whether individual T. castaneum larvae show

91 induction of preference for four novel resources, given temporal heterogeneity in resource availability

92 (sequential exposure to alternative resources). Second, we quantified the induction of preference when two

93 resources were thoroughly mixed and presented simultaneously. Third, we quantified the induction of preference

94 under spatial resource heterogeneity, wherein alternative resources were presented to the larvae in separate

95 patches and larvae were free to experience both resources by moving between patches. Finally, we tested the

96 hypothesis that induction of resource preference is adaptive for larvae.

\section{METHODS}

\section{Beetle stock maintenance and experimental individuals}

100 We generated an outbred laboratory population from 12 wild-collected populations of T. castaneum from across

101 India. We maintained the population at $34^{\circ} \mathrm{C}\left( \pm 1^{\circ} \mathrm{C}\right)$ in $750 \mathrm{~g}$ whole-wheat flour (ancestral resource; henceforth 102 "wheat") on a 35 day discrete-generation cycle with 2500 to 5000 individuals per generation. T. castaneum is a

103 generalist and can consume a wide variety of resources. We conducted our assays using the ancestral resource

104 for our population - whole-wheat flour (abbreviated 'W') - and four novel resources: finger millet flour

105 (Eleusine coracana, 'FM'), sorghum flour (Sorghum bicolor, 'S'), refined wheat flour ("maida" flour, 'RW') and

106 rice flour (Oryza sativa, 'R'). These are among the most common flours used in India; hence, T. castaneum is

107 likely to encounter them in Indian storage warehouses. T. castaneum larvae perform best in their ancestral

108 resource, wheat flour (Figure S1). They show significantly higher mortality in rice flour ( 23\% drop in survival

109 compared to wheat), and significantly delayed development in rice and finger millet flours ( $>100 \%$ and 37\%

110 delay in development rate respectively, relative to wheat) (Figure S1).

112 We derived all experimental individuals from generations 12-23 of the outbred stock population. To generate

113 experimental individuals, we allowed 250-400 randomly picked stock adults (7-14 days post-eclosion) to

114 oviposit in 150 g of finely sifted wheat flour for 24 hours. We collected eggs and placed each egg in a well of 48 - 
115 well polystyrene plates (Sigma Costar; hereafter "treatment plates"), with $\sim 1 \mathrm{~g}$ of the appropriate resource per

116 egg. We placed all plates inside a dark incubator maintained at $34^{\circ} \mathrm{C} \pm 1{ }^{\circ} \mathrm{C}$ while larvae developed. Each 48 -

117 well plate contained replicate eggs for a single resource treatment. After 14 days of larval development, we

118 tested the resource choice of each larva as described below. We tested larval choice on the $15^{\text {th }}$ day of

119 development since at this stage larvae can be easily handled and observed, but still need to feed for a few days

120 before pupating (hence their resource choice at this stage could have fitness consequences). For each experiment

121 described below, we aimed to test an equal number of individuals per treatment. However, for various reasons

122 (e.g. an egg did not hatch, a larva was accidentally killed during handling), the actual sample size differed

123 slightly across treatments. For clarity, we provide the sample size for each treatment in the relevant figures in the

124 main text and supplementary information.

125

\section{Testing larval resource choice}

127 We measured larval resource choice in petri-plates containing adjacent patches of two resources. We spread $\sim 0.8$

128 g resource on each half of a $60 \mathrm{~mm}$ petri-plate in a thin layer, with no space between the resources except for a

129 circular area in the center of the plate (17 mm diameter) where we placed the 15 day old larva with no bias in

130 orientation (Figure S2A). This placement ensured that each larva had the opportunity to sample both resources

131 presented in the choice test. We noted the position of each larva every 12 hours ( \pm 1 hour) for 48 hours. All

132 larvae were housed in a dark incubator at $34^{\circ} \mathrm{C}\left( \pm 1^{\circ} \mathrm{C}\right)$, and were outside the incubator for $<2$ hours when we

133 transferred larvae into new resources (see below) or while we noted larval position during the choice assay. For

134 each larva, we calculated preference for the novel resource as the percent occurrence in the new resource patch.

135 Larval tracks visible from the bottom of the petri-plate showed that larvae were capable of exploring the entire

136 plate in a 12 hour time period. For all our experiments, these tracks confirmed that almost all larvae explore both

137 resources, even if they showed an extreme preference for either resource. This suggests that larval movement

138 across time points was not constrained by patch size. 
140 Initially, we collected data on larval position for 72 hours. However, we found that some larvae from each

141 treatment pupated towards the end of this period. Since pupae are non-feeding, data collected close to pupation

142 may be unreliable with respect to feeding choice. For larvae that did not pupate in 72 hours, we found that larval

143 preference calculated after 48 hours was well correlated (Spearman correlation coefficient $>0.8$ ) with preference

144 calculated from 72 hours of data (Figure S3). Hence, for subsequent experiments, we only collected 48 hours of

145 data on larval position (4 data points per larva). On average, $<1 \%$ of the larvae pupated during the 48 hour

146 period across all treatments, and these were excluded from subsequent analysis.

148 Our assay assumes that larval presence in a resource patch indicates foraging in that patch, and that time spent

149 foraging in a patch is proportional to the time spent feeding in that patch. If the larva was not visible during

150 readings, we used a pair of forceps to gently move through the resource patches until the larva was found. If the

151 larva was found in contact with both resource patches during a reading, it was noted as being present in both

152 resources. Outside of the duration of larval transfers into new resources and the reading during the choice assay,

153 all larvae were held in a dark incubator at $34^{\circ} \mathrm{C}\left( \pm 1^{\circ} \mathrm{C}\right)$. Since the resources we used have different textures, we

154 measured differences in larval mobility in each resource to account for resource-specific movement rates. We did

155 this by measuring the number of times larvae moved across patches of the same resource in a similar setup

156 (Figure S2B). Larval movement rate was similar across resources, suggesting that slower larval movement in

157 novel resources does not confound our measure of larval preference for the novel resource (Figure S4).

158

\section{Analyzing data to test for induction of larval preference}

160 To test the impact of temporal resource heterogeneity on the occurrence and magnitude of induction of

161 preference, we exposed larvae to alternative resources either sequentially or simultaneously. To measure

162 induction, we compared the resource preference of test larvae (given prior experience with a novel resource) to

163 the preference of control larvae that had never experienced the novel resource (i.e. that had developed for 14

164 days only in wheat). A significantly higher preference for the new resource in test vs. control larvae would 
165

166

167

168

169

170

171

172

173

174

175

176

177

178

179

180

181

182

183

184

185

186

187

188

189

190

indicate induction of preference. For example, if rice patch occupancy of control larvae was 20\%, but was

$\sim 60 \%$ for larvae exposed to rice during development, this would indicate an induced preference for rice.

We conducted all statistical analyses in R (R core team, 2016) using RStudio (RStudio team, 2015). For each

experimental treatment measuring larval resource choice, we had position data for four time points per

individual. We measured preference for a novel resource as patch occupancy, i.e. the proportion of times (out of

4 measured) that a larva was observed in the novel resource. Since our measurements uncover strong and

repeatable patterns of preference across blocks (not reported here) and we find that larvae frequently explore

both patches within a 12-hour time window, this measure reflects true preference rather than sequential auto-

correlation between time points. We used a generalised linear model with binomial errors and a logit link

function to test whether patch occupancy differed significantly between control larvae and test larvae from the same experiment, unless mentioned otherwise. The odds ratio calculated from the fitted model coefficients is

reported as the fold increase in patch occupancy in a treatment relative to the control. For example, a three-fold increase in preference (i.e. patch occupancy) for a novel resource in a treatment indicates that the larvae in this treatment were three times more likely than the control larvae to choose the novel resource.

\section{Testing preference induction with temporal resource heterogeneity}

To test whether induction of resource preference depends on the timing of experience with a resource, we changed the resource available to larvae at different time points during development. For instance, to test the effect of early vs. late experience, we allowed larvae to experience a novel resource only during the first or the second week of development. The remaining development was completed in the ancestral resource. For this experiment, we placed eggs in a specific resource for the first week of development (as described above). To change larval exposure to a resource, we pooled week-old replicate individuals from a treatment plate and sifted them out of the resource using a fine sieve. The first resource was shaken off from their body during the sifting process. We used a fine brush to place each larva into a well of a clean 48-well plate containing the second resource. For treatments that required exposure to a single resource throughout larval development, we followed 
191 the same procedure at the 1-week time point, but placed larvae in fresh resource of the same type. We used this

192 protocol to test the impact of larval experience with distinct resources at various time points. Therefore, we

193 measured the impact of early (only first week of development), late (only second week of development) or

194 continuous experience (both weeks of development) with four novel resources, on larval preference for the novel 195 resource.

197 To test the impact of larval experience with finger millet immediately prior to the choice assay, we followed the above protocol, but allowed larvae to develop in the first resource for 13 days and then transferred them to the second resource for 1 day. On the following day (day 15), we measured larval preference as above. Therefore, we had four treatments - larvae that only experienced either wheat or finger millet throughout (but were transferred on day 13 to control for the impact of transfer), larvae that developed for 13 days in either wheat (or

\section{Testing specificity of the induced preference}

205 We tested whether larval preference induced by a particular resource was specific to the experienced resource 206 rather than a generalized acceptance of any new resource. We allowed larvae to develop in one of three 207 treatments for 14 days: no exposure to finger millet, second week exposure to finger millet, or complete 208 development in finger millet. On the $15^{\text {th }}$ day, we gave each larva a choice between the ancestral resource 209 (wheat) and one of the four novel resources (finger millet, sorghum, refined wheat and rice). An increased 210 preference only for finger millet would indicate that the induced preference is specific to the experienced 211 resource and is not a general increased acceptance of any novel resource. Another possibility is that experience 212 with a novel resource increases repulsion from the ancestral resource, which may be interpreted as increased

213 preference for the novel resource in our assays. To test this, we allowed larvae to develop in one of four

214 treatments for 14 days: no exposure to finger millet, only first week or second week exposure to finger millet, 215 and complete development in finger millet. On the $15^{\text {th }}$ day, we gave larvae a choice between two novel 216 resources: sorghum and finger millet. If exposure to finger millet did not increase repulsion from wheat, then 
217 larvae would still choose finger millet in this assay. These two experiments allowed us to confirm that the

218 induction of preference is indeed a specific increased preference for the experienced resource, rather than

219 repulsion from the ancestral wheat resource.

\section{Testing preference induction with homogeneous resource mixes}

222 To measure the impact of simultaneous experience with two resources on larval preference, we weighed out 223 equal amounts of two resources (wheat and finger millet). We mixed these together so that larvae would not be 224 able to choose between the two resources while feeding (as in Parent et al. 2014). We collected eggs from stock 225 beetles and allowed larvae to hatch and develop individually for 14 days in pure wheat, pure finger millet

226 ("FM"), or the homogeneous mix (50\% finger millet by weight) of the two resources. In three separate 227 experiments, we then tested the preference of larvae from each of these treatments, given the following choice 228 combinations - wheat versus finger millet, wheat versus the $50 \%$ mix, or the $50 \%$ mix versus finger millet. To

229 test whether larvae were able to distinguish between small differences in finger millet concentrations in a

230 homogeneous mix, we allowed larvae to experience the 50\% homogeneous FM-wheat mix and gave them the

231 following choices: 25\% FM (in wheat, as above) versus 50\% FM; 75\% FM versus 50\% FM; and 25\% FM versus

232 75\% FM. After experiencing a 50\% mix, if larvae preferred it over other concentrations (such as 25\% FM and

233 75\% FM), this would demonstrate very high specificity of the induced preference. We confirmed that none of the

234 resource mixes decreased larval survival, though the 75\% FM mix did delay larval development (Figure S1).

\section{Testing preference induction with spatial heterogeneity in resource availability}

237 To measure larval preference induction in a spatially heterogeneous patchy habitat, we directly placed eggs from

238 stock individuals in a two-patch habitat containing the ancestral resource and a novel resource. To create the 239 patchy habitat, we spread a thin layer of $\sim 1 \mathrm{~g}$ resource on either half of $60 \mathrm{~mm}$ petri-plates. We placed an egg in 240 one patch of this habitat with a fine brush, and allowed it to develop undisturbed for 14 days (one egg per petri241 plate). During development, larvae were free to move across patches and consume the resource of their choice. 
242 After 14 days, we noted the position of each larva as an overall indicator of larval preference during

243 development and tested its resource preference as described above.

245 Estimating the impact of larval choice on larval fitness

246 To determine the fitness consequences of experience-mediated larval resource choice, we collected eggs from

247 the stock population and measured larval survival and development rate under conditions of temporal

248 heterogeneity in resource availability. We exposed larvae to wheat or finger millet during the first, second and

249 third week of development, alternating between the two resources. Larvae in control groups experienced

250 homogeneous conditions and experienced either wheat or finger millet throughout (but were transferred to fresh

251 flour each week). This experimental design mimicked larval resource use under spatial as well as temporal

252 heterogeneity, if larvae acted on their resource preference. For example, while exploring a heterogeneous habitat,

253 if a larva experienced finger millet and increasingly preferred it to wheat, it would consume largely finger millet

254 during the remainder of its development. Larvae from the wheat-finger millet-finger millet treatment would

255 represent this scenario, allowing us to determine the fitness impact of induced preference for finger millet

256 relative to larvae that never experienced finger millet (i.e. the wheat-wheat-wheat controls). Note that if we

257 measured larval fitness in a patchy habitat, we would not be able to determine which resource larvae had

258 consumed, and would thus be unable to connect preference to fitness. After three weeks of development, we

259 counted the number of surviving larvae and noted their developmental stage. A higher proportion of adults

260 and/or pupae would indicate faster development, which is typically beneficial for early reproduction and reduced

261 probability of larval parasitism (A. Fred West 1960; Blaser \& Schmid-Hempel 2005). We used a generalised

262 linear model with binomially distributed errors and a logit link function to test whether the proportion of live

263 individuals and proportion of pupae or adults out of all live individuals were significantly different across

264 treatments.

265

266 RESULTS

267 Induction of larval preference for new resources is time-sensitive and resource-specific 
268 We tested whether prior experience with four new resources - finger millet, sorghum, refined wheat and rice

269 flour - could elicit an induction of preference for these resources. We exposed larvae to one of four treatments -

270 no exposure to the novel resource (control), exposure either during the first or second week of development, and

271 exposure throughout development. We found that prior experience with a new resource typically increased larval

272 preference for that resource, compared to the baseline preference shown by control larvae (Figure 1, Table S1).

273 However, this induction of preference occurred only when larvae were exposed to the novel resource during the

274 second week of development. In contrast, when early experience with a new resource was followed by a week in

275 wheat flour, larvae did not show an increased preference for the novel resource. The only exception to these

276 patterns was rice flour. Both baseline and induced preference for rice flour were very low, indicating that larvae

277 are extremely averse to rice (Figure 1D). In contrast to the results for other resources, larvae exposed to rice

278 flour during the first week of development showed a reduced preference for rice, although the effect size was

279 very low (Figure 1D, Table S1).

281 Overall, the magnitude of the induction of preference varied across resources, with a greater than six-fold

282 increase in preference for finger millet (Figure 1A) but a modest two-fold increase for sorghum (Figure 1B) and

283 refined wheat (Figure 1C) (Table S1). We found that an individual's sex did not significantly affect the

284 magnitude of preference induction (Figure S6). Interestingly, longer exposure to the new resource (for 2 weeks)

285 also did not alter the effect size of preference induction, except for a decrease in preference for finger millet with

286 longer exposure (compare the last two bar plots in Figure 1A; Table S1). For the following experiments, we

287 focused on finger millet flour, which showed the largest effect size for induction of preference.

289 As described above, recent ( $2^{\text {nd }}$ week only) but not early ( $1^{\text {st }}$ week only) experience with a new resource induced

290 preference for the resource. However, it was unclear whether induction of preference was contingent on the

291 developmental stage (i.e. the second week of larval development) or on exposure to the novel resource

292 immediately prior to the preference test. Thus, we tested whether reducing the period of later experience could 
293 still induce preference. We found that even one day of exposure to finger millet immediately before the choice

294 test was sufficient to induce preference, albeit with a decrease in the effect size (Figure 2, Table S2). Notably,

295 one day of exposure to wheat was also enough to counter the effects of previous exposure to finger millet for

296 thirteen days (Figure 2). These results strongly suggest that recent exposure to finger millet, rather than exposure

297 during a specific developmental stage, is critical for induction of larval preference for finger millet.

299 Next, we tested whether the induction of larval preference for finger millet flour is specific to finger millet, or is wheat flour (ancestral resource) or attraction to finger millet. After the larva had experienced finger millet, we offered it a choice between finger millet and sorghum. We found that the induction of preference for finger millet was intact in this novel context, demonstrating that aversion to wheat flour does not explain the induction of preference observed in finger-millet flour. Taken together, our results demonstrate a specific, experiencemediated increase in preference for novel resources in flour beetle larvae (Figure S8, Table S4).

\section{Simultaneous experience with two resources also elicits specific induction of larval preference}

311 In the above experiments, we presented larvae with alternative resources in a temporally distinct manner (i.e. 
319 presented with the two pure resources in the choice test showed a strong preference for pure finger millet flour

320 (Figure 3A). These results suggest that the presence of the ancestral resource does not qualitatively interfere with

321 preference induction for the novel resource, although the effect size was about half that observed for induction

322 with pure finger millet (Figure 3A; Table S5A). Interestingly, larvae reared on a 1:1 finger millet-wheat mix did

323 not distinguish between mixes with different proportions of finger millet and wheat. For example, when given a

324 choice between the 1:1 mix versus a $25 \%$ or a $75 \%$ mix, larvae that developed in the 1:1 mix did not show a

325 preference for the experienced mix (Figure S9). Together, these results show that even half the amount of finger

326 millet experienced simultaneously with wheat can induce preference for finger millet; but larvae are unable to

327 distinguish quantitatively between resources mixes with different proportions of finger millet.

329 Impact of spatial habitat heterogeneity on induction of preference

330 Next, we tested whether a spatially heterogeneous habitat can alter larval preference via induction. We placed

331 individual eggs in one patch of a two-patch habitat containing the ancestral wheat resource, and either finger-

332 millet ("FM") flour or refined wheat ("RW") as a new resource. Larvae developed while moving freely across

333 patches, consuming their resource of choice. After 15 days, we found that in the FM-wheat habitat, larvae

334 typically occupied the same resource patch where they were placed as eggs (i.e. the natal resource; Figure 4A).

335 Regardless of where they were placed as eggs, larvae that developed in the patchy FM-wheat habitat showed a

336 consistently higher probability of choosing finger millet in the choice test ( 40\%; Figure 4B). Thus, prior contact

337 with the finger millet even during exploration of the habitat was sufficient to induce a preference for finger

338 millet. In contrast, in the RW-wheat habitat, most individuals that were initially placed in the refined wheat patch

339 moved to the wheat patch over the course of development (Figure 4A). However, larvae initially placed in

340 refined wheat did show a significantly higher preference for refined wheat compared to individuals placed in the

341 wheat patch as eggs (Figure 4C). In this habitat, it appears that the natal resource induced larval preference.

342 Hence, the relative use of the natal resource in a patchy habitat varies depending on the alternative resource

343 available in that habitat. 


\section{Induction of preference can be maladaptive for larval development}

346 What are the fitness consequences of larval preference induction? Earlier, we found that larvae that developed in

347 a new resource did not pay a mortality cost, except in rice where larval mortality was significantly higher than in

348 wheat ( 65\% survival in rice, $\sim 80 \%$ in wheat; Figure S1). Finger millet and rice were both suboptimal for larval

349 development rate (Figure S1). Of the four tested resources, the only resource that did not induce preference was

350 rice flour, which is also the only resource that substantially decreases larval survival. However, we found that

351 across resources, the magnitude of preference induction was not associated with larval development rate in those 352 resources.

354 In the experiments described above, we measured the fitness consequences of consuming a single resource 355 throughout larval development. To simulate the fitness consequences of varying larval resource choice in a 356 heterogeneous habitat, we transferred the larvae to a different resource for three developmental windows of one 357 week each. This represents a scenario in which larvae move across resource patches every week. Thus, we could estimate the fitness consequences of larvae exerting preference and consuming different preferred resources over the course of their development. For example, to estimate the development rate of larvae that experienced finger millet in the second week of development in a heterogeneous habitat (containing wheat and finger millet

361 patches), we allowed larvae to develop in wheat for one week, finger millet for the second, and wheat for the 362 third. After allowing larvae to develop in these regimes, we measured the fraction of larvae that successfully 363 pupated or eclosed as adults as a fitness proxy.

We found that regardless of the treatment, nearly all larvae pupated within three weeks, and had similar rates of survival (Figure S10). Of the larvae that developed in wheat for the first two weeks of development, 10\% had also completed the pupal stage and eclosed as adults (Figure 5). However, almost none of the larvae that 


\section{DISCUSSION}

373 Despite a large body of work on the induction of larval preference, we understand very little about the ecological

choice. We demonstrate that $T$. castaneum larvae fed on their ancestral wheat resource prefer wheat, but are more resource choice and altering their fitness in complex environments.

Our finding of resource-specific effect size of preference induction corroborates results from earlier studies with 
397

specific compound(s) used by larvae for food recognition (del Campo et al. 2001) varies across resources, altering short-term recognition of these resources. The induction of preference with recent, brief experience (1 day), and the observed resource specificity are both consistent with this hypothesis. Therefore, we speculate that the chemical composition of the different resources may determine the strength of preference induction.

An important feature of our experimental system was our ability to test the impact of experience with simultaneously experienced alternative resources. In phytophagous insects such as Lepidopterans that directly feed on plants, it is difficult to present a mix of different resources at the same time. A priori, we expected that experiencing a novel resource as part of a resource mix should weaken the induction of preference, compared to experiencing a pure novel resource. Our findings are consistent with this expectation. We observe that larvae discriminate not only between pure resources but also between a homogenous mix of these resources, preferring exactly what they experienced when it is available. This suggests that when two resources are experienced simultaneously in a mix, a strong preference is induced for a mix of these resources, over a pure form of either. Interestingly, larvae that experienced a balanced mix of a novel resource (finger millet) and the ancestral resource (wheat) strongly preferred $100 \%$ finger millet over $100 \%$ wheat. A simple expectation is that larvae should not discriminate between wheat and finger millet, having experienced an equal amount of both during development. In contrast, our results suggest that different components in a single mix have quantitatively different effects on larval preference, with the ancestral resource in the mix impacting larval preference much less than an equal mass of the novel resource. Thus, some novel resources (in this case, finger millet) can induce larval preference more strongly than the ancestral resource, indicating an asymmetry in the impact of different resources on insect preference. A similar phenomenon was observed in Manduca sexta larvae, where across choice combinations, acceptable non-host plants induced a stronger preference than host plants (Boer 1992). However, in this study, alternative hosts were presented separately and preference was not measured using choice assays. Interestingly, we found that the specificity of induced resource preference did not extend to the exact concentrations of the experienced mix (Figure S9). Our data do not distinguish between the alternate possibilities of whether larvae are incapable of distinguishing between different concentrations of resources, or 
423 whether they do not discriminate between them. While experience with homogeneous mixes is probably not very

424 common in nature, our data hint at the underlying mechanism of induction of preference in T. castaneum larvae,

425 and are consistent with the hypothesis of additive resource-specific modifications in the sensory system leading

426 to increased preference for a resource mix. However, we need further investigation of the exact mechanism of

427 the induction of preference (Bernays \& Weiss 1996; Anderson \& Anton 2014).

429 An intriguing and novel finding from our work is that resource-specific induction of preference can also occur in

430 a spatially heterogeneous habitat. Importantly, in this experiment, we found that larvae are capable of moving

431 out of their natal resource, thereby impacting their own fitness. An earlier study with fruit flies also showed that

432 larvae could move away from a suboptimal natal habitat chosen by their mother (Abbott \& Dukas 2016).

433 However, in our experiments, larvae did not move away from finger millet, even though it significantly delays

434 larval development. On the other hand, larvae avoided refined wheat, which confers similar larval survival and

435 development rate as wheat. Similarly, previous work showed that flour beetle larvae reared in a wheat-corn

436 patchy habitat preferred the suboptimal corn resource more strongly than larvae reared in a homogeneous wheat

437 habitat (Parent et al. 2014). Our current results suggest that this suboptimal preference for corn may have been

438 induced by prior exposure to corn. Together, these observations indicate that alternative resources experienced at

439 a small spatial scale may alter larval preference, and possibly the fitness of developing larvae. Thus, our

440 experiments provide new insights about the sensitivity of larval preference to their natal environment and nearby

441 alternative resources and underscore the importance of studying larval preference in heterogeneous habitats.

443 By explicitly measuring larval resource preference, movement and fitness, we showed that larval resource use is

444 strongly driven by resource-specific induction rather than the potential fitness consequences of their choice. On

445 the one hand, the lack of induction with rice flour may be seen as adaptive, since larval survival is significantly

446 lower in rice compared to the other resources (all of which showed significant preference induction; Figure S1).

447 On the other hand, larval development rate was not correlated with the strength of preference induction across

448 resources. For example, larval preference was most strongly induced by finger millet, a resource that decreases 
449 development rate substantially. These results are similar to previous findings in Manduca sexta, where the

450 strength of larval preference induction was not correlated with larval performance across hosts (Silva et al.

451 2014). If beetle larvae acted on induced preference during early development, they would suffer a significant

452 developmental delay ( 10-18\% lower eclosion rate at three weeks) compared to larvae that did not consume

453 finger millet. Given these fitness consequences, optimally behaving larvae should move out of a finger millet

454 patch, particularly during the first two weeks of development. However, our data show that this does not occur in

455 a patchy habitat, possibly due to the strong induction by finger millet. Taken together, our experiments suggest

456 that the induction of resource preference may be maladaptive, and may serve as a barrier to optimal resource

457 choice in heterogeneous habitats.

458

459 Is the induction of larval resource preference broadly relevant in insects? In the specific case of flour beetles,

460 habitat heterogeneity and resource turnover is likely a common feature in stored grain warehouses, particularly

461 where the parent populations used in this study were collected. However, we could not systematically measure

462 temporal or spatial heterogeneity in the natural habitat of Tribolium castaneum, and we hope that future studies

463 can quantify such heterogeneity. More generally, if insect larvae explore their habitats, they may often encounter

464 local heterogeneity in patch quality (as in Abbott \& Dukas 2016; Soler et al. 2012; Gamberalle-Stille et al.

465 2014). Our data suggest that larval resource use in such heterogeneous habitats can be driven by several features

466 of the habitat, such as spatial and temporal heterogeneity in resource availability. In this context, our experiments

467 provide new insights about the sensitivity of larval preference to their environment and underscore the

468 importance of studying larval preference in heterogeneous environments. Larval resource choice may also play

469 an important role in the context of suboptimal maternal oviposition. A growing body of evidence suggests

470 several constraints on optimal female oviposition in generalists (Charlery de la Masselière et al. 2017), such as

471 search time, patch complexity, and the ability to receive and process sensory information (Janz \& Nylin 1997;

472 Mayhew 1997; Bernays 1999; Bernays \& Funk 1999; Bernays 2001; Janz 2002). Thus, studying only

473 oviposition preference in such habitats may not reliably predict larval resource use, and alternative resources

474 experienced at a smaller spatial scale may alter the fitness outcomes for developing larvae. 
In closing, we note that many questions regarding the ecological and evolutionary consequences of larval resource choice remain unanswered. This is primarily because larval choice has been underrepresented in studies in non-Lepidopteran species may help us develop general principles of insect host choice and understand its

481 implications for individual fitness. Experience based plasticity in larval resource choice may be an important mechanism through which rapid behavioural diet shifts can occur, either when female oviposition behaviour is suboptimal, or under conditions of habitat heterogeneity where larval resource availability fluctuates. Under such conditions, the induction of preference may enhance larval performance on suboptimal resources through increased feeding (Agrawal et al. 2002b). We find that induction of preference does not occur for a resource that substantially decreases larval survival. Therefore, this may well be a mechanism through which rapid niche expansion can occur within the larval stage of individuals, altering subsequent acceptability of the novel resource and allowing individuals to utilize and adapt to novel resources (West-Eberhard 1989). Preference induction may thus facilitate local adaptation in populations experiencing heterogeneity in resource availability.

490 Our study represents the first systematic analysis of the impact of habitat heterogeneity on larval preference for 491 new resources, and suggests its potential role in the evolution of diet breadth.

\section{Acknowledgements}

494 We thank members of the Agashe lab and Shannon Olsson for discussion and comments on the manuscript. This 495 work was supported by the National Centre for Biological Sciences and an INSPIRE Faculty award to DA (IFA49613 LSBM-64).

498 Author contributions

499 DA, VRK and SI designed experiments; VRK and SI conducted experiments; VRK, SI and DA analyzed data;

500 VRK and DA wrote the manuscript. All authors gave final approval for publication. 


\section{References}

503

504

505

506

507

508

509

510

511

512

513

514

515

516

517

A. Fred West, Jr., 1960. The biology of a species of Nosema (Sporozoa : Microsporidia) parasitic in the flour beetle Tribolium confusum. The Journal of Parasitology, 46(6), pp.747-754.

Abbott, K.R. \& Dukas, R., 2016. Substrate choice by ovipositing mothers and consequent hatchling behaviour: the exploration sharing hypothesis. Animal Behaviour, 121, pp.53-59. Available at: http://dx.doi.org/10.1016/j.anbehav.2016.08.012.

Abisgold, B.Y.J.D. \& Simpson, S.J., 1988. The effect of dietary protein levels and haemolymph composition on the sensitivity of the maxillary palp chemoreceptors of locusts. Journal of Experimental Biology, 135, pp.215-229.

Agashe, D. \& Bolnick, D.I., 2012. Dietary niche and population dynamic feedbacks in a novel habitat. Oikos, 121(3), pp.347-356.

Agosta, S.J., 2006. On ecological fitting, plant-insect associations, herbivore host shifts, and host plant selection. Oikos, 114(3), pp.556-565.

Agrawal, A.A., Vala, F. \& Sabelis, M.W., 2002. Induction of preference and performance after acclimation to novel hosts in a phytophagous spider mite : adaptive plasticity? American Naturalist, 159(5), pp.553-565.

Anderson, P. \& Anton, S., 2014. Experience-based modulation of behavioural responses to plant volatiles and other sensory cues in insect herbivores. Plant, Cell and Environment, pp.1826-1835.

Berdegue, M., Reitz, S.R. \& Trumble, J.T., 1998. Host plant selection and development in Spodoptera exigua : do mother and offspring know best ? Entomologia Experimentalis et Applicata, 89, pp.57-64.

Berdegue, M. \& Trumble, J.T., 1996. Effects of plant chemical extracts and physical characteristics of Apium graveolens and Chenopodium murale on host choice by Spodoptera exigua larvae. Entomologia Experimentalis et Applicata, 78, pp.253-262.

Bernays, E.A., 2001. Neural limitations in phytophagous insects : implications for diet breadth and evolution of host affiliation. Annual Review of Entomology, 46, pp.703-727.

Bernays, E.A., 1999. When host choice is a problem for a generalist herbivore : experiments with the whitefly, Bemisia tabaci. Ecological Entomology, pp.260-267.

Bernays, E.A. \& Funk, D.J., 1999. Specialists make faster decisions than generalists : experiments with aphids. Proceedings of the Royal Society of London B, 266(1999), pp.151-156.

Bernays, E.A. \& Weiss, M.R., 1996. Induced food preferences in caterpillars: the need to identify mechanisms. Entomologia Experimentalis et Applicata, 78, pp.1-8.

Blaser, M. \& Schmid-Hempel, P., 2005. Determinants of virulence for the parasite Nosema whitei in its host Tribolium castaneum. Journal of invertebrate pathology, 89, pp.251-257. 
Boer, G. de, 1992. Diet-induced food preference by Manduca sexta larvae: acceptable non-host plants elicit a stronger induction than host plants. Entomologia Experimentalis et Applicata, 63, pp.3-12. Available at: http://doi.wiley.com/10.1111/j.1570-7458.1992.tb02414.x.

de Boer, G. \& Hanson, F.E., 1984. Foodplant selection and induction of feeding preference among host and nonhost plants in larvae of the tobacco hornworm Manduca sexta. Entomologia Experimentalis et Applicata, 35(2), pp.177-193.

Campbell, J.F. \& Hagstrum, D.W., 2002. Patch exploitation by Tribolium castaneum : movement patterns, distribution and oviposition. Journal of Stored Products Research, 38, pp.55-68.

del Campo, M. \& Renwick, J.A.A., 2000. Induction of host specificity in larvae of Manduca sexta : chemical dependence controlling host recognition and developmental rate. Chemoecology, 10, pp.115-121.

del Campo, M.L., Miles, C.I., Schroeder, F.C., Mueller, C., Booker, R., Renwick, J.A., 2001. Host recognition by the tobacco hornworm is mediated by a host plant compound. Nature, 411, pp.186-189.

Carlsson, M.A., Anderson, P., Hartlieb, E., Hansson, B., 1999. Experience-dependent modification of orientational response to olfactory cues in larvae of Spodoptera littoralis. Journal of Chemical Ecology , 25(11), pp.2445-2454.

Charlery de la Masselière, M., Facon B., Hafsi, A., Duyck, P., 2017. Diet breadth modulates preference performance relationships in a phytophagous insect community. Scientific Reports, 7(16934), p.1-9. Available at: http://www.nature.com/articles/s41598-017-17231-2.

Chew, F.S., 1977. Coevolution of pierid butterflies and their cruciferous foodplants II - The distribution of eggs on potential foodplants. Evolution, 31(3), pp.568-579.

Dethier, V.G., 1959. Egg-laying habits of Lepidoptera in relation to available food. The Canadian Entomologist, 91(9), pp.554-561

Gamberale-Stille, G.,Soderlind, L., Janz, N. and Nylin, S., 2014. Host plant choice in the comma butterfly-larval choosiness may ameliorate effects of indiscriminate oviposition. Insect Science, 21(4), pp.499-506.

Glendinning, J.I., Ensslen, S., Eisenberg, M.E., Weiskopf, P., 1999. Diet-induced plasticity in the taste system of an insect : Localization to a single transduction pathway in an identified taste cell, The Journal of Experimental Biology, 202, pp.2091-2102.

Gretes, W.C., Stanwyck, E.A. \& Hanson, F.E., 2016. Innate and acquired components of oligophagy in the herbivorous lepidopteran, Manduca sexta. Entomologia Experimentalis et Applicata, 160(3), pp.259-271.

Gripenberg, S., Mayhew, P.J., Parnell, M., Roslin, T., 2010. A meta-analysis of preference-performance relationships in phytophagous insects. Ecology Letters, 13, pp.383-393.

Hagstrum, D.W. \& Gilbert, E.E., 1976. Emigration rate and age structure dynamics of Tribolium castaneum populations during growth phase of a colonizing episode. Environmental Entomology, 5(3), pp.445-448.

Henniges-Janssen, K., Heckel, D.G. \& Groot, A.T., 2014. Preference of diamondback moth larvae for novel and original host plant after host range expansion. Insects, pp.793-804. 
Jaenike, J., 1978. On optimal oviposition behavior in phytophagous insects, Theoretical Population Biology, 14(3), pp.350-356.

Janz, N., 2002. Evolutionary ecology of oviposition strategies. In : Hilker, M. and Meiners, T., editors Chemoecology of insect eggs and egg deposition,Blackwell Verlag GmbH, pp.349-376.

Janz, N. \& Nylin, S., 1997. The role of female search behaviour in determining host plant range in plant feeding insects : a test of the information processing hypothesis. Proceedings of the Royal Society B, pp.701-707.

Jermy, T., Hanson, F. \& Dethier, V.G., 1968. Induction of specific food preference in Lepidopterous larvae. Entomologia Experimentalis et Applicata, 11, pp. 211-230.

Karowe, D.N., 1989. Facultative monophagy as a consequence of prior feeding experience : behavioral and physiological specialization in Colias philodice larvae. Oecologia, 78, pp.106-111.

Mayhew, P.J., 1997. Adaptive patterns of host-plant selection by phytophagous insects. Oikos, 79(3), pp.417428. Available at: http://www.jstor.org/stable/3546884.

Mayhew, P.J., 2001. Herbivore host choice and optimal bad motherhood. Trends in Ecology and Evolution, 16(4), pp.165-167.

Naylor, A.F., 1961. Dispersal in the red flour beetle Tribolium castaneum (Tenebrionidae). Ecology, 42(2), pp.231-237.

Parent, C.E., Agashe, D. \& Bolnick, D.I., 2014. Intraspecific competition reduces niche width in experimental populations. Ecology and Evolution, pp.3978-3990.

Phillips, W.M., 1977. Modification of feeding "preference” in the flea beetle, Haltica lythri (Coleoptera, Chrysomelidae). Entomologia Experimentalis et Applicata, 21(1), pp.71-80. Available at: http://doi.wiley.com/10.1111/j.1570-7458.1977.tb02658.x.

Schoonhoven, L.M., 1967. Loss of hostplant specificity by Manduca sexta after rearing on an artificial diet. Entomologia Experimentalis et Applicata, 10, pp.270-272.

Schoonhoven, L.M. \& Meerman, J., 1978. Metabolic costs of changes in diet and neutralization of allelochemicals, 24, pp.489-493.

Scriber, J.M., 1979. The effects of sequentially switching foodplants upon biomass and nitrogen utilization by polyphagous and stenophagous Papilio larvae. Entomologia Experimentalis et Applicata, 25(1979), pp.203-215.

Silva, A.K., Lopes Goncalves, G. \& Rudinei Pires Moreira, G., 2014. Larval feeding choices in heliconians : induced preferences are not constrained by performance and host plant phylogeny. Animal Behaviour, 89, pp.155-162. Available at: http://dx.doi.org/10.1016/j.anbehav.2013.12.027.

Singer, M.C. \& McBride, C.S., 2010. Multitrait, host-associated divergence among sets of butterfly populations: implications for reproductive isolation and ecological speciation. Evolution, 64(4), pp.921-933.

Sokoloff, A.,1974. The Biology of Tribolium, with Special Emphasis on Genetic Aspects, Vol. 2. Oxford University Press, Oxford, UK. 
604 Soler, R., Pineda, A., Li, Y., Ponzio, C., J. A. van Loon, J., Weldegergis, B.T., Dicke M., 2012. Neonates know 605 better than their mothers when selecting a host plant. Oikos, 121(12), pp.1923-1934.

606 West-Eberhard, M.J., 1989. Phenotypic plasticity and the origins of diversity. Annual Review of Ecology and $607 \quad$ Systematics, 20, pp.249-278.

608 Wiklund, C., 1975. The evolutionary relationship between adult oviposition preferences and larval host plant 609 range in Papilio machaon L ., Oecologia , 18, pp.185-197.

610 Yamamoto, R.T., 1974. Induction of hostplant specificity in the tobacco hornwork, Manduca sexta. Journal of Insect Physiology, 20, pp.641-650.

612 Zhou, D.-S., Wang, C.-Z. \& Loon, J.J.A. van, 2009. Chemosensory basis of behavioural plasticity in response to 613 deterrent plant chemicals in the larva of the small cabbage white butterfly Pieris rapae, Journal of Insect $614 \quad$ Physiology, 55, pp.788-792.

615 Ziegler, J.R., 1977. Dispersal and reproduction in Tribolium : The influence of food level. Journal of Insect $616 \quad$ Physiology, 23, pp.955-960.

617 Ziegler, J.R., 1976. Evolution of the migration response : emigration by Tribolium and the influence of age.

618 Evolution, 30, pp.579-592. 


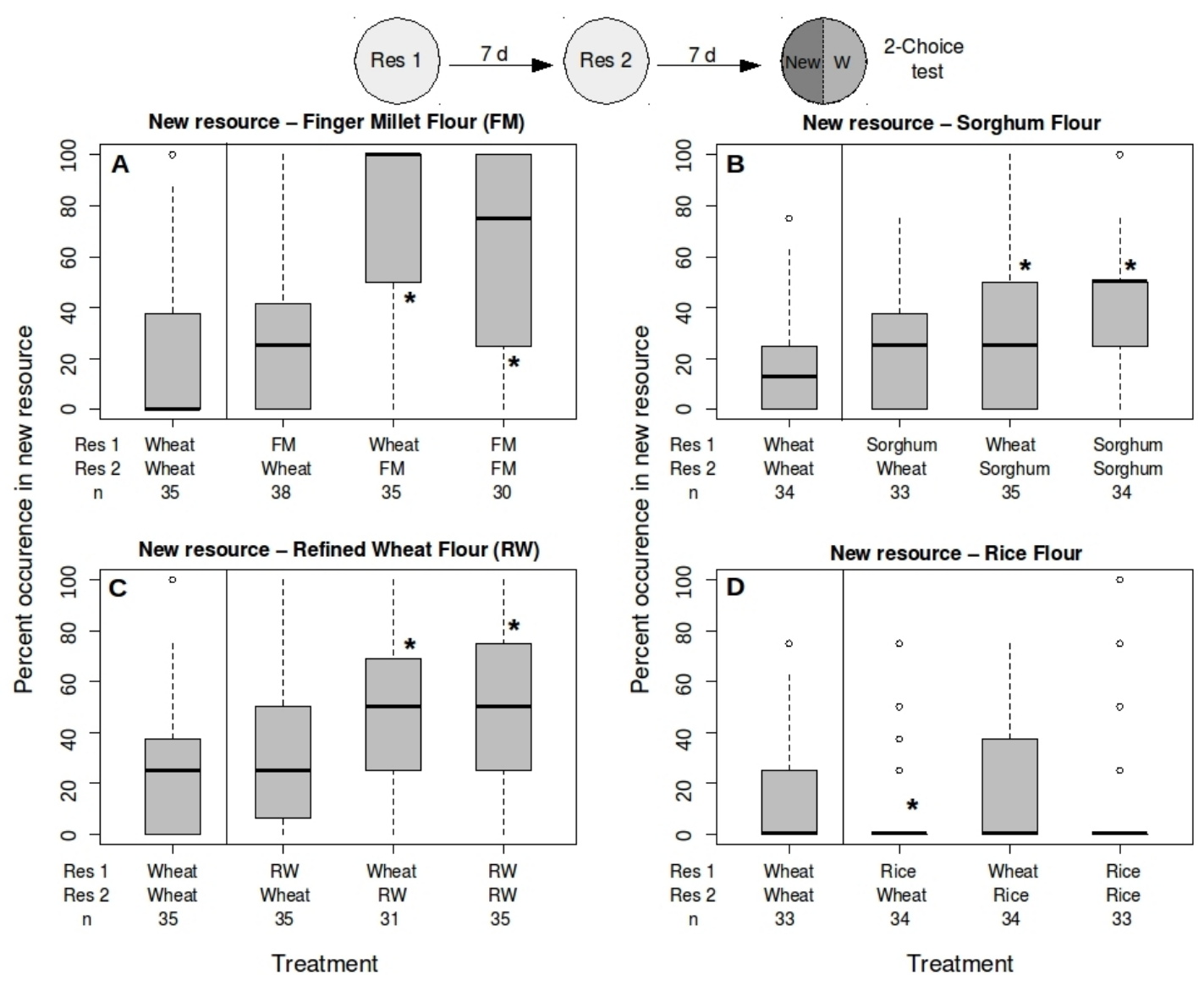

619 Figure 1: Induction of larval resource preference with two different resources presented sequentially

620 during development. Each panel shows preference of single larvae for a novel resource when given a choice

621 between the ancestral resource (wheat, "W") and the novel resource for 48 hours. Percent occurrence in a novel

622 resource was calculated based on 4 readings of larval patch occupancy. The novel resources in each panel are (A)

623 finger millet flour (B) sorghum flour (C) refined wheat flour (D) rice flour. Larvae were reared in resource 1 for

624 the first week, and resource 2 in the second week (x-axis). In each panel, the left bar represents a control

625 treatment where larvae were never exposed to the novel resource until the choice test. Numbers below each

626 boxplot indicate sample size. Asterisks indicate treatments that differ from the control $(\mathrm{p}<0.05$, generalized

627 linear model with binomially distributed errors). Boxplots indicate median and first and third quartiles, and

628 whiskers indicate the range of data. All figures with boxplots follow this convention. 
631

632

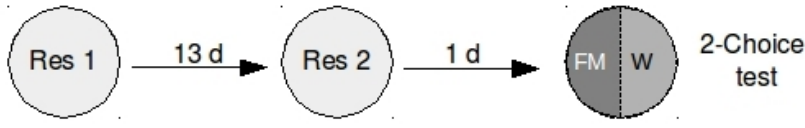

633

Novel resource - Finger Millet (FM) Flour

634

635

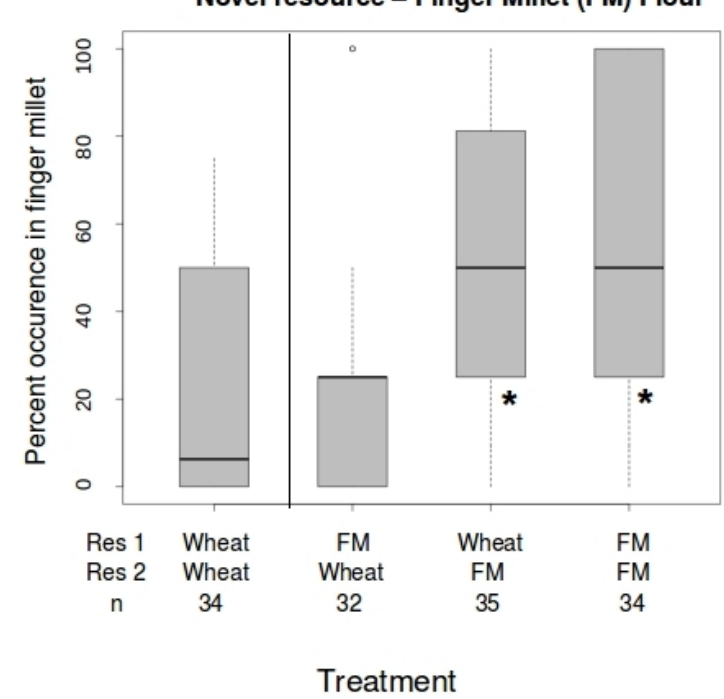

Figure 2: Induction of preference for finger millet

occurs in a short time. Larvae were reared in resource

1 for 13 days and then resource 2 for 1 day (x-axis).

Larval preference was measured as described in Fig 1.

Boxplots represent data as described in Figure 1. 


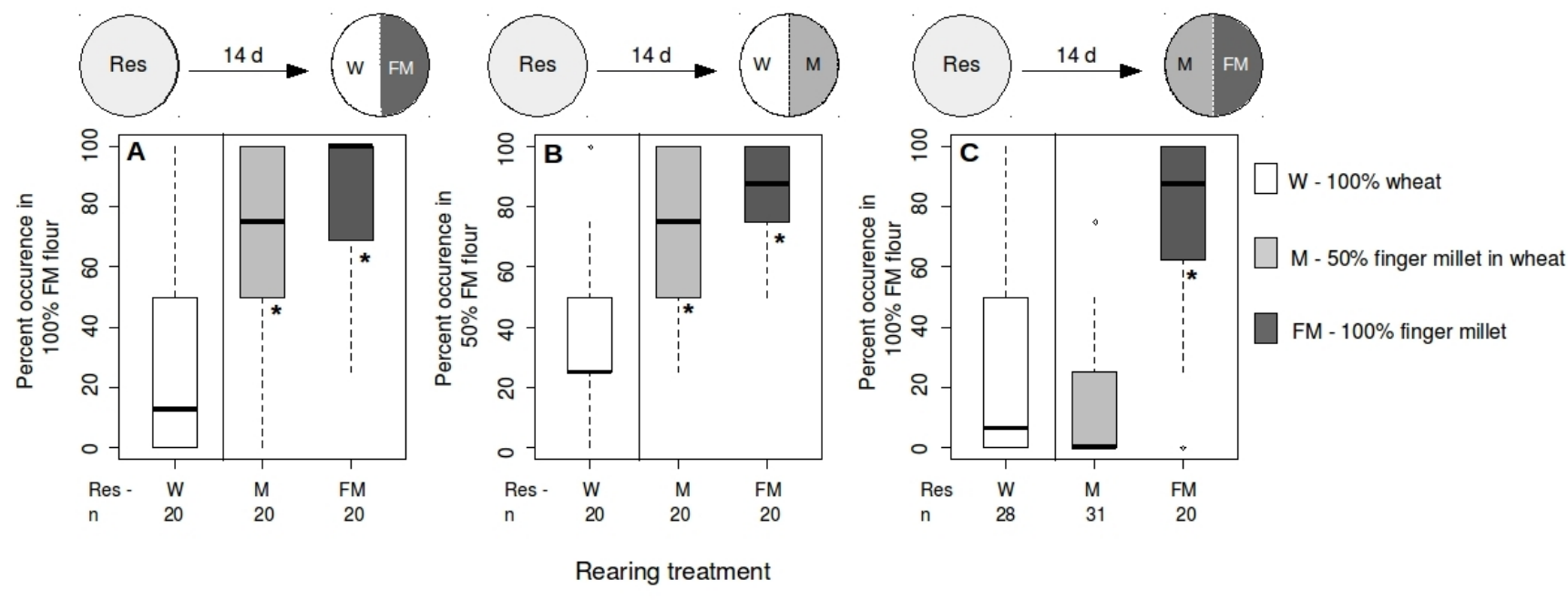

638 Figure 3: Induction of preference also occurs with a mixture of finger millet and wheat flour. Each panel

639 shows preference of single larvae for a resource mix with the higher concentration (by mass) of finger millet

640 flour when given a choice between various combinations among 100\% wheat flour (white), 1:1 mix of finger

641 millet flour and wheat (light grey) and 100\% finger millet flour (dark grey) for 48 hours. Larval preference was

642 measured as described in Figure 1. The choice combinations in each panel are (A) 100\% wheat versus 100\%

643 finger millet (B) 100\% wheat versus 50\% finger millet and (C) 50\% finger millet versus 100\% finger millet.

644 Larvae were reared in each treatment (x-axis) for 14 days. Boxplots represent data as described in Figure 1. 

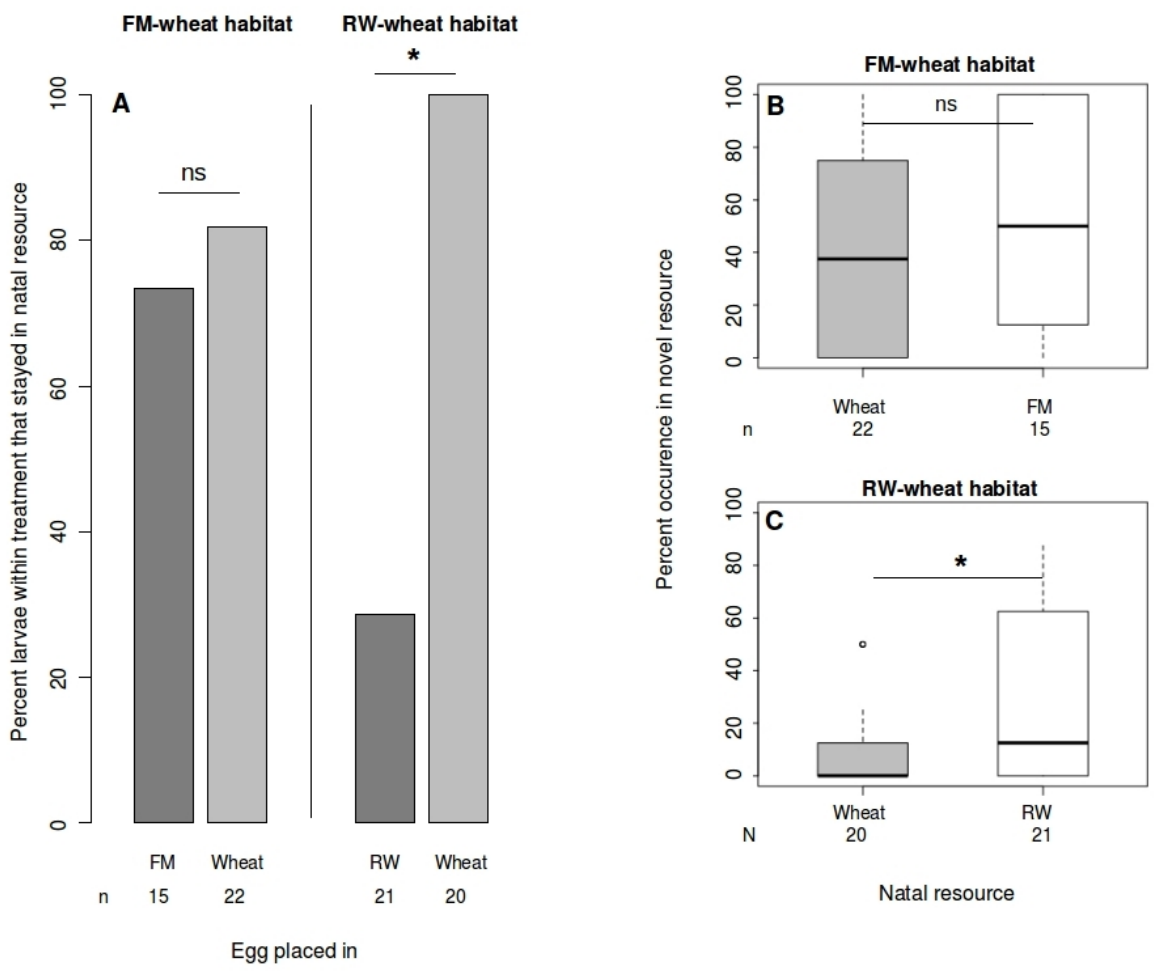

646 Figure 4: Larval preference for new resources after development in a heterogeneous two-patch habitat.

647 Individual eggs were placed in one patch of a two-patch heterogeneous habitat, and larvae were allowed to

648 develop allowing for unrestricted movement across patches. (A) Percentage of larvae found in the same patch

649 they hatched into (natal resource) in either an FM-wheat (left) or an RW-wheat (right) patchy habitat after 14

650 days of development. Asterisks indicate a significant difference between the indicated treatments $(\mathrm{p}<0.05$,

651 pairwise two-sided chi-squared test of proportions with Yates' continuity correction). The new resource in

652 subsequent preference tests on the $15^{\text {th }}$ day was either (B) finger millet (FM) or (C) refined wheat (RW) flour.

653 Larvae within each treatment are split along the $\mathrm{x}$-axis according to their natal resource. The $\mathrm{y}$-axis denotes

654 larval preference for the natal resource. Asterisks indicate a significant difference in percent occurrence in the

655 new resource across individuals in different natal resources. $(\mathrm{p}<0.05$, generalised linear model with binomially

656 distributed errors). Boxplots represent data as described in Figure 1. 
657
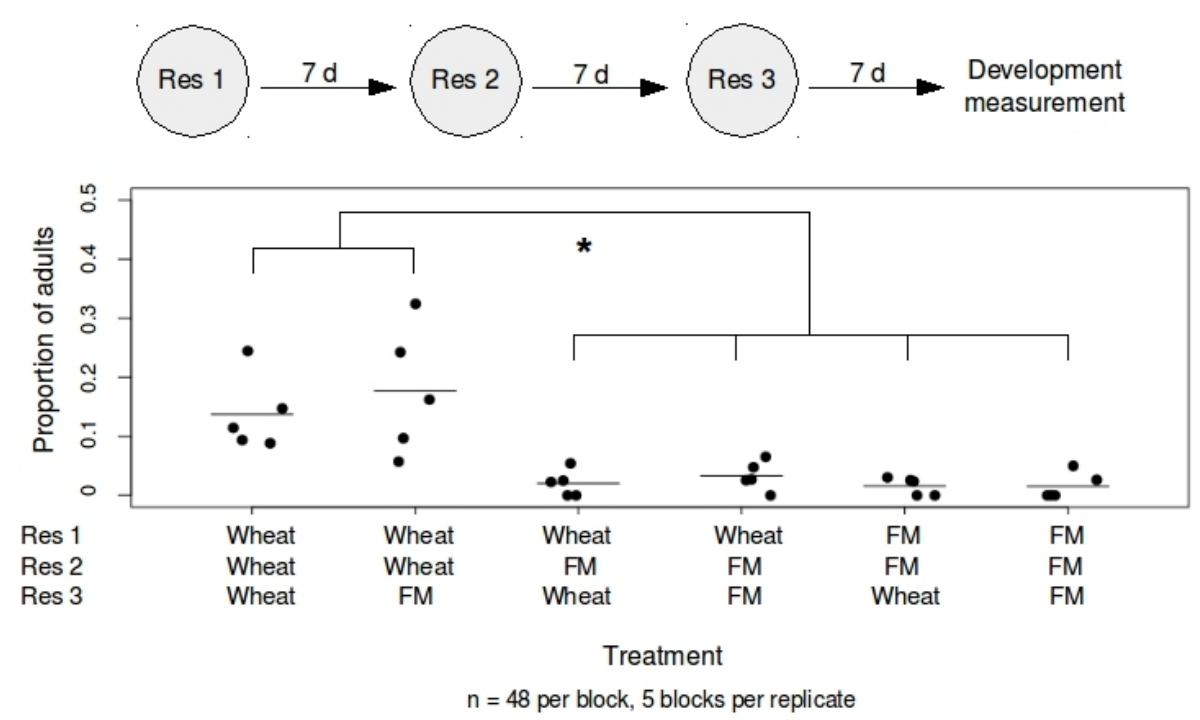

658 Figure 5: Development rate of larvae as a function of their respective developmental experience. Each

659 point represents data for a single experimental block. Within each block, individual eggs (n = 48 per

660 developmental regime) were placed in each of the treatments represented along the $\mathrm{x}$-axis. The $\mathrm{y}$-axis represents

661 the proportion of individuals that had eclosed as adults on the $21^{\text {st }}$ day. Lines within the scatter represent mean of

662 the data. Asterisks indicate significant difference in development rate between treatments as represented (p <

663 0.05, generalized linear model with binomially distributed errors) 